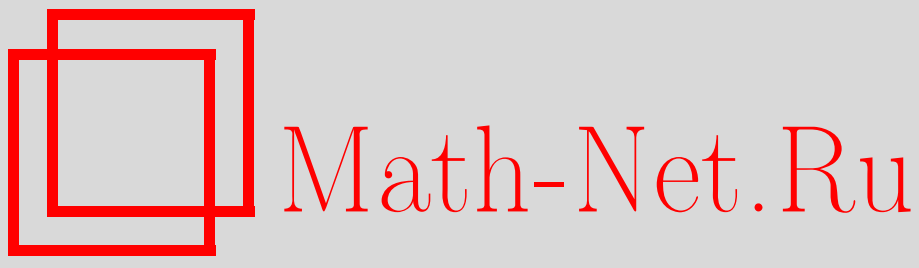

В. П. Радченко, Д. В. Шапиевский, О дрейфе упругой деформации для нелинейноупругих материалов вследствие ползучести, Вестн. Сам. гос. техн. ун-та. Сер. Физ.-мат. науки, 2006, выпуск 43, 99-106

DOI: https://doi.org/10.14498/vsgtu458

Использование Общероссийского математического портала Math-Net.Ru подразумевает, что вы прочитали и согласны с пользовательским соглашением

http://www. mathnet.ru/rus/agreement

Параметры загрузки:

IP: 34.239 .49 .27

26 апреля 2023 г., 06:49:01

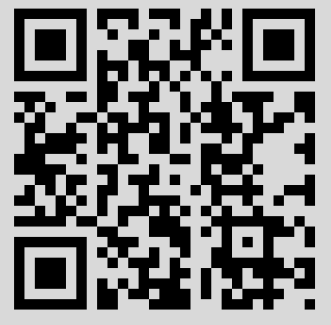




\section{О ДРЕЙФЕ УПРУГОЙ ДЕФОРМАЦИИ ДЛЯ НЕЛИНЕЙНО-УПРУГИХ МАТЕРИАЛОВ ВСЛЕДСТВИЕ ПОЛЗУЧЕСТИ}

На основе моделирования нелинейно-упругого материала структурной моделью средь показано, что вследствие ползучести мгновенно-упругая деформация существенно зависит от накопленной деформации ползучести. В результате дрейфа упругой деформации вследствие ползучести ее величина после разгрузки может быть как больше, так и меньше аналогичной величины при нагрузке. В начальный момент времени доказан ряд теорем и следствия из них, детализирующих рассматриваемые эффекты.

Многие природные и промышленные материалы представляют собой сложную систему, состоящую из множества кристаллических зерен, цепочек молекул, размеры, форма и ориентация которых имеют случайный характер. Поэтому наряду с феноменологическими теориями, устанавливающими связь между макроскопическими свойствами материала, возникает необходимость (особенно для сред со сложными реологическими свойствами) рассматривать микромеханизмы деформирования и разрушения материала с целью более полного описания его поведения при термомеханических воздействиях. Это дает представление о том, каким образом формируются макроскопические характеристики материала, и позволяет более обоснованно выбрать подходящий вариант феноменологической теории.

В этом направлении особое место занимают структурные математические модели среды, в которых неоднородное микронапряженное состояние моделируется так называемой конструкционной неоднородностью. Согласно этому подходу материал представляется совокупностью некоторых гипотетических элементов, наделенных простейшими свойствами упругости, пластичности и вязкого течения [1-8], при этом основное внимание в этих работах уделялось линейно-упругим средам. Однако некоторые природные биокомпозитные материалы обладают нелинейным законом упругости и для них наблюдаются сложные эффекты влияния деформации ползучести на упругую деформацию, которые с феноменологических позиций сложно понять и описать [9-11]. Целью настоящей работы является изучение и анализ отмеченных эффектов с позиций механики микронеоднородных сред.

1. Предполагается, что сплошная среда моделируется совокупностью гипотетических локальных элементов. Уравнения состояния каждого $i$-того локального элемента описывается следующими кинетическими уравнениями:

$$
\begin{aligned}
& y^{i}(t)=\varphi\left(\eta^{i}(t), x^{i}(t)\right), \varphi^{i}(0,0)=0 ; \\
& \dot{\phi}=f^{i}\left(\eta^{i}(t), x^{i}(t)\right), \eta^{i}(0)=0 ; i=1,2, \ldots, l .
\end{aligned}
$$

Здесь $x^{i}(t)=\left(x_{1}^{i}(t), x_{2}^{i}(t), \ldots, x_{m_{i}}^{i}(t)\right)$ - вектор-функция нагрузок на локальный элемент. Координатами вектора $x^{i}(t)$ в зависимости от математической природы локальных элементов могут быть микронапряжения $\sigma_{\eta \omega}^{i}$, приложенные усилия, значения температуры и т.п. Вектор $y_{i}(t)=\left(y_{1}^{i}(t), y_{2}^{i}(t), \ldots, y_{n_{i}}^{i}(t)\right)$ - вектор-функция, описывающая деформационные свойства локального элемента (микронапряжения, микроперемещения и т.д.); $\eta_{i}(t)=\left(h_{1}^{i}(t), h_{2}^{i}(t), \ldots, h_{s_{i}}^{i}(t)\right)-$ вектор-функция состояния локального элемента, при помощи которой фиксируется предыстория процесса деформирования локального элемента; $f^{i}$ и $\varphi^{i}$ - вектор-функции $m_{i}+s_{i}$ переменных с $n_{i}$ и $s_{i}$ координатами соответственно.

Введем вектор нагрузок $q=\left(q_{1}, q_{2}, \ldots, q_{m}\right)$, приложенный к материальной точке макросреды (макронапряжения, усилия, моменты и т.д). Поскольку локальные элементы взаимодействуют друг с другом, то к уравнениям состояния (1) необходимо добавить $k_{1}$ уравнений равновесия и $k_{2}-$ уравнений совместности деформаций локальных элементов:

$$
\begin{gathered}
F_{i_{1}}^{1}\left(x_{1}, x_{2}, \ldots, x_{l}, q_{1}, q_{2}, \ldots, q_{m}\right)=0, i_{1}=1,2, \ldots, k ; \\
F_{j_{1}}^{2}\left(y_{1}, y_{2}, \ldots, y_{l}\right)=0, \quad j_{1}=1,2, \ldots, k_{2},
\end{gathered}
$$


где $k_{1}+k_{2}=\sum_{i=1}^{l} m_{i}=m_{0}$.

Система уравнений (1)-(3) описывает некоторую математическую структурную модель материала, при этом $m_{0}$ - число внутренних, а $m$ - внешних степеней свободы модели. Равенства (2), (3) отражают структуру математической модели, а уравнения (1) — свойства её отдельных локальных элементов.

Для описания макродеформационных характеристик среды вводится в рассмотрение вектор $Y=\left(Y_{1}, Y_{2}, \ldots, Y_{N}\right)$, координатами которого могут быть макродеформации, макроперемещения и т.д. Очевидно, что должна существовать функциональная зависимость, связывающая микро- и макродеформированные состояния:

$$
\begin{gathered}
Y_{i}=\Psi_{i}\left(y_{1}, y_{2}, \ldots, y_{l}\right) ; \\
\Psi_{i}(0,0, \ldots, 0)=0, i=1,2, \ldots, N,
\end{gathered}
$$

где $\Psi_{i}$ - вектор-функция с $m_{0}$ координатами. В механике деформируемого твердого тела для построения соотношений (4) используются различные гипотезы, в частности гипотезу однородности деформаций по объему, различные методы осреднения микродеформаций и т.д.

Так как координаты вектора $y^{i}=\left(y_{1}^{i}, y_{2}^{i}, \ldots, y_{n_{i}}^{i}\right)$ связываются с микродеформационными характеристиками, то при малых деформациях справедливо $\left|y_{j}^{i}\right|=1$ ( $\left.j=1,2, \ldots, n_{i}\right)$. Предполагая далее, что функции $\Psi_{i}$ непрерывно дифференцируемы, разложим (4) в ряд Тейлора и ограничимся линейными членами. Тогда с учетом (5) получим

$$
Y_{k}=\sum_{i=1}^{l} \sum_{j=1}^{n_{i}} \frac{\partial \Psi^{k}}{\partial y_{j}^{i}} y_{j}^{i}, \quad k=1,2, \ldots, N .
$$

Конкретизируем соотношения (1) применительно к задачам ползучести. Практический интерес вызывает случай, когда часть координат вектор-функции $y^{i}=\varphi^{i}\left(\eta^{i}, x^{i}\right)$ зависит лишь от $x^{i}(t)$, а другая часть - от $\eta^{i}(t)$. Тогда первое соотношение (1) можно представить в виде

$$
y^{i}=\left(\varphi_{1}^{i}\left(\eta^{i}\right), \varphi_{2}^{i}\left(x^{i}\right)\right), \varphi_{1}^{i}(0)=0, \varphi_{2}^{i}(0)=0
$$

где $\varphi_{1}^{i}$ и $\varphi_{2}^{i}$ - вектор-функции с $n_{1}^{i}$ и $n_{2}^{i}$ координатами, причем $n_{1}^{i}+n_{2}^{i}=n_{i}, 0 \leq n_{1}^{i} \leq s$, $0 \leq n_{2}^{i} \leq m_{i}$.

Как следует из второго соотношения (1) и (7), координаты $\varphi_{1}^{i}$ изменяются непрерывно, а $\varphi_{2}^{i}$ - мгновенно при ступенчатом изменении $x(t)$ на конечную величину. Отсюда следует, что координатами $\varphi_{2}^{i}$ могут являться, например, упругие, температурные, пластические деформации, а координатами $\varphi_{1}^{i}$ - деформации ползучести (или ее компоненты).

Гипотеза отделимости (7), введенная Ю. П. Самариным в [12], позволяет существенно конкретизировать структуру соотношения (1) для локального элемента.

ТЕОРЕМА 1. Если для определяющего уравнения локального элемента (1) выполняется гипотеза отделимости (7), вектор-функции $\varphi_{1}^{i}$ и $\varphi_{2}^{i}$ непрерывно дифференцируемы и имеют отличные от нуля якобианы по некоторым $n_{1}^{i} u n_{2}^{i}$ переменным (соответственно), то при $s<\infty$ уравнения (1) приводятся к виду

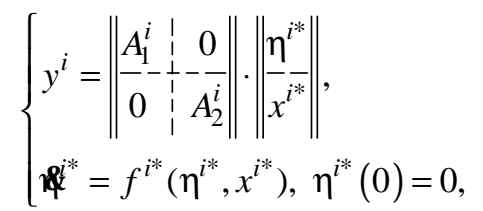

где $\eta^{i^{*}}=\eta^{i^{*}}\left(\eta^{i}\right), x^{i^{*}}=x^{i^{*}}\left(x^{i}\right)$ - столбцуовые $s_{1} \times 1$ и $m_{i} \times 1$ матрицьи, $A_{1}^{i}$ и $A_{2}^{i}$ - постояннье $n_{1}^{i} \times s_{i}$ и $n_{2}^{i} \times m_{i}$ матрицы, причем $\operatorname{rang} A_{1}^{i}=n_{1}^{i}, \operatorname{rang} A_{2}^{i}=n_{2}^{i}$. 
В работе [12] аналогичная теорема доказана для некоторого управляемого объекта и поэтому приведенное там доказательство можно формально перенести на теорему 1.

В дальнейшем будем считать, что определяющие соотношения (1) для локального элемента уже приведены к виду (8) и звездочки для всех функций, кроме $x^{i^{*}}$, будем опускать, т.е. в качестве определяющих уравнений для локального элемента будем использовать соотношения

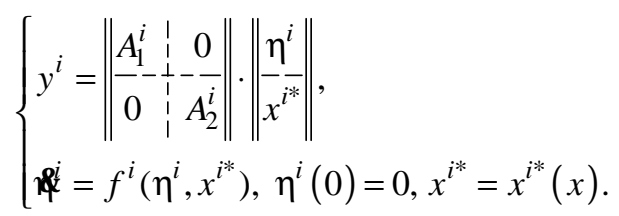

Таким образом, структурная модель описывается уравнениями (2), (3), (9).

Подставляя первое соотношение (9) в (3) и решая полученную систему уравнений (2), (3), находим функции

$$
x^{i^{*}}=\Phi^{i}\left(q_{1}, \ldots, q_{m}, \eta^{1}, \ldots, \eta^{l}\right), i=1,2, \ldots l
$$

(соответствующий якобиан системы должен быть отличен от нуля).

Уравнения (10) замыкают систему уравнений (9), так как внешние воздействия $q_{j}(t)$ $(j=1,2, \ldots, m)$ предполагаются известными.

Установим связь макродеформационных характеристик вектора $Y$ с вектором $q=\left(q_{1}, q_{2}, \ldots, q_{m}\right)$. Для этого подставим $y^{i}$ из первого соотношения (9) в соотношение (6):

$$
Y_{k}=\sum_{i=1}^{l} \sum_{j=1}^{n_{i}} b_{i j}^{k}\left(\sum_{p=1}^{s_{i}} a_{1 . p j}^{i} \eta_{p}^{i}+\sum_{p=1}^{m_{i}} a_{2 . p j}^{i} x_{p}^{i^{*}}\right), k=1,2, \ldots, N,
$$

где $b_{i j}^{k}=\frac{\partial \Psi^{k}}{\partial y_{j}^{i}}$ (см. соотношения (6)).

В конечном итоге соотношение (11) можно представить в виде

$$
\begin{gathered}
Y=Y^{e}+Y^{p} ; \\
Y^{e}=C_{1} x^{*}, Y^{p}=C_{2} \eta,
\end{gathered}
$$

где $C_{1}$ и $C_{2}$ - постоянные $N \times m_{0}$ и $N \times s \quad\left(m_{0}=\sum_{i=1}^{l} m_{i}, s=\sum_{i=1}^{l} s_{i}\right)$ постоянные матрицы; $x^{*}(x)$, $\eta$ - столбцовые $m_{0} \times 1$ и $s \times 1$ матрицы.

Первое соотношение (13) описывает мгновенно изменяемые слагаемые вектора $Y$ (упругие, пластические, температурные и другие макрохарактеристики), а второе - реологические слагаемые $Y$ (деформации ползучести, перемещения от ползучести и т.п.). Используя уравнения (9), (10), (12), (13), можно установить связь между макрохарактеристиками среды в точке (материальном объеме) и внешними воздействиями $q=\left(q_{1}, q_{2}, \ldots, q_{m}\right)$ :

$$
\begin{gathered}
\left\{\begin{array}{l}
Y=C_{1} x^{*}+C_{2} \eta, \\
\psi=f^{*}\left(\eta, x^{*}\right) ;
\end{array}\right. \\
x^{*}=\Phi^{*}(q, \eta),
\end{gathered}
$$

где $\Phi^{*}-$ вектор-функция из $l$ переменных с $m+s$ координатами.

Система уравнений (14), (15) позволяет определить макродеформационные характеристики среды, связывая значения $Y_{k}$ с вектором внешних воздействий $q$, и может служить обоснованием традиционного макроскопического (феноменологического) подхода к описанию деформирования материала, когда соответствующие определяющие уравнения строятся без анализа микронапряженного состояния материала и сразу формируется связь между тензорами напряжений и деформаций.

Таким образом, в общем случае при построении феноменологических реологических уравнений нужно учитывать, что, как это следует из (14), (15), мгновенно изменяемые (в частности упругие) координаты вектора $Y$ могут зависеть от реологических координат вектора состояний $\eta$. Это обуславливает дрейф мгновенно изменяемых координат за счет реологических эффектов. По внешнему проявлению это явление аналогично изменению упругой деформации 
при постоянном напряжении в процессе старения [13], однако, как это будет показано ниже, механизм этого явления совершенно другой и реализуется лишь для нелинейно-упругих материалов $[14,15]$. Следует отметить, что на возможность такого эффекта для статически неопределимых конструкций впервые было указано Ю. П. Самариным в работе [16] и несколько позже проиллюстрировано в работе [17] .

2. Для выяснения условий, приводящих к эффекту зависимости мгновенно-упругой деформации от реологической деформации, рассмотрим случай одноосной ползучести материала при постоянной температуре. Детальный анализ опубликованных в литературе экспериментальных данных показал, что этот эффект проявляется лишь у природных биокомпозитных материалов (компактной костной ткани) $[10,11]$ с ярко выраженными свойствами нелинейности для упругой деформации $[9,18]$. С целью конкретизации полученных выше результатов для математического моделирования таких сред в качестве структурной модели материала возьмем $l$ параллельно соединенных локальных элементов типа обобщенной модели Максвелла, наделенных свойствами нелинейной упругости и нелинейной вязкости, так, что полная деформация каждого элемента $\varepsilon_{i}$ представляется в виде

$$
\begin{gathered}
\varepsilon_{i}=e_{i}+p_{i}, \\
e_{i}=\varphi\left(\sigma_{i}(t)\right), p_{i}(t)=A_{i}\left(\sigma_{i}(t)\right), i=1,2, \ldots, l,
\end{gathered}
$$

где $e_{i}$ - упругая деформация; $p_{i}$ - деформация ползучести; $A_{i}$ - некоторый временной (в общем случае - нелинейный) оператор; $\sigma_{i}$ - микронапряжение в локальном элементе; $\varphi_{i}(0)=0, p(0)=0$.

Обозначим через $\sigma(t)$ макронапряжение, а через $\varepsilon(t)$ - макродеформацию. Запишем уравнение равновесия для структурной модели

$$
\sum_{i=1}^{l} \alpha_{i} \sigma_{i}(t)=\sigma(t)
$$

и (в предположении гипотезы однородности деформаций по объему) уравнения совместности деформаций

$$
\varepsilon_{i}(t)=\varepsilon(t), i=1,2, \ldots, l,
$$

где $\alpha_{i}$ - «вес» локального элемента с номером $\left.i, \alpha_{i} \in\right] 0 ; 1\left[, \sum_{i=1}^{l} \alpha_{i}=1\right.$.

Пусть при $t \in] 0 ; t^{*}$ [ на образец действует постоянное макронапряжение $\sigma(t)=\sigma_{0}$, которое снимается при $t=t^{*}\left(\sigma(t)=0\right.$ при $\left.t>t^{*}\right)$. Задача состоит в изучении обратимости упругой деформации образца, которая возникает при $t=+0$ и должна исчезнуть после разгрузки.

С помощью соотношений (16)-(19) для определения начальных напряжений при $t=+0$ получаем систему уравнений

$$
\begin{gathered}
\sum_{i=1}^{l} \alpha_{i} \sigma_{i}^{0}=\sigma_{0} ; \\
\varphi_{1}\left(\sigma_{1}^{0}\right)=\varphi_{2}\left(\sigma_{2}^{0}\right), \quad i=2,3, \ldots l,
\end{gathered}
$$

где $\sigma_{i}^{0}=\sigma_{i}(+0), i=1,2, \ldots, l$.

В результате ползучести образца при $\sigma(t)=\sigma_{0}$ к моменту разгрузки значения микронапряжений $\sigma_{i}^{*}=\sigma_{i}\left(t^{*}\right)$ согласно (16)-(19) удовлетворяют системе уравнений:

$$
\begin{gathered}
\sum_{i=1}^{l} \alpha_{i} \sigma_{i}^{*}=\sigma_{0} \\
\varphi_{1}\left(\sigma_{1}^{*}\right)+p_{1}^{*}=\varphi_{i}\left(\sigma_{i}^{*}\right)+p_{i}^{*}, \quad i=2,3, \ldots l,
\end{gathered}
$$

где $p_{i}=p_{i}\left(t^{*}\right)$.

После разгрузки при $t=t^{*}$ в локальных элементах возникнут остаточные напряжения $\Delta \sigma_{i}=$ $=\sigma_{i}\left(t^{*}+0\right)$, удовлетворяющие системе уравнений:

$$
\sum_{i=1}^{l} \alpha_{i} \Delta \sigma_{i}=0
$$




$$
\varphi_{1}\left(\Delta \sigma_{1}\right)+p_{1}^{*}=\varphi_{i}\left(\Delta \sigma_{i}\right)+p_{i}^{*}, i=2,3, \ldots, l .
$$

Согласно гипотезе (19) упругая макродеформация при нагрузке $e(0)=\varphi_{1}\left(\sigma_{1}^{0}\right)$, а при разгрузке в момент $t=t^{*}-e\left(t^{*}\right)=\varepsilon\left(t^{*}-0\right)-\varepsilon\left(t^{*}+0\right)=\varphi_{1}\left(\sigma_{1}^{*}\right)-\varphi_{1}\left(\Delta \sigma_{1}\right)$.

Очевидно, что упругая макродеформация будет полностью обратимой, если выполняется условие

$$
\varphi_{1}\left(\sigma_{1}^{0}\right)=\varphi_{1}\left(\sigma_{1}^{*}\right)-\varphi_{1}\left(\Delta \sigma_{1}\right)
$$

ТЕОРЕМА 2. Если локальные элементы структурной модели (16)-(19) удовлетворяют линейному закону упругости $\varphi_{i}(\sigma)=\frac{\sigma}{E_{i}} \quad(i=1,2, \ldots, l)$, где $E_{i}-$ микромодуль Юнга, то упругая макродеформация не зависит от деформаџии ползучести и условие (26) выполняется.

Д о к а з а т е л в с т в о. С учетом конкретизации функции $\varphi_{i}$ из (21) имеем

$$
\sigma_{i}^{0}=\sigma_{1}^{0} \frac{E_{i}}{E_{1}}
$$

Подставляя (27) в (20) и решая полученное уравнение относительно $\sigma_{1}^{0}$, получим

$$
\varphi\left(\sigma_{1}^{0}\right)=\frac{\sigma_{1}^{0}}{E_{1}}=\frac{\sigma_{0}}{\sum_{i=2}^{l} \alpha_{i} E_{i}} .
$$

С учетом (22), (24) будет справедливым равенство

$$
\sum_{i=1}^{l} \alpha_{i}\left(\sigma_{i}^{*}-\Delta \sigma_{i}\right)=\sigma_{0}
$$

Вычитая из каждого равенства (23) соответствующие равенства (25), с учетом конкретизации $\varphi_{i}$, находим

$$
\sigma_{i}-\Delta \sigma_{i}=\frac{E_{i}}{E_{1}}\left(\sigma_{1}^{*}-\Delta \sigma_{i}\right), i=2,3, \ldots, l .
$$

Подставляя (30) в (29) и решая полученное уравнение относительно $\sigma_{1}^{*}-\Delta \sigma_{1}$, получим

$$
\varphi\left(\sigma_{1}^{*}\right)-\varphi\left(\Delta \sigma_{1}^{*}\right)=\frac{\left(\sigma_{1}^{*}-\Delta \sigma_{1}\right)}{E_{1}}=\frac{\sigma_{0}}{\sum_{i=2}^{l} \alpha_{i} E_{i}} .
$$

Из (28), (31) очевидно, что условие (26) выполняется. Теорема доказана.

Таким образом, если локальные элементы структурной модели (16)-(19) (и материал) следуют линейным законам упругости, то при любых законах ползучести локальных элементов (и материала) упругая деформация не зависит от деформации ползучести и феноменологические реологические уравнения для материала можно строить, отдельно анализируя упругую и реологическую компоненты. Этот факт подтверждается обширными экспериментальными исследованиями по ползучести материалов различной природы (металлы, пластмассы и др.).

ТЕОРЕМА 3. Если хотя бы один локальный элемент структурной модели (16)-(19) следует нелинейному закону упругости, то соотношение (26) не выполняется и величина упругой деформации материала зависит от накопленной деформаџии ползучести.

Д о к а з а т е л ь с т в о. Предположим для простоты, что первые $l-1$ локальных элементов удовлетворяют линейному, а $l$-тый элемент - нелинейному степенному закону упругости, т.е.

$$
\varphi_{i}(\sigma)=\frac{\sigma}{E_{i}}(i=1,2, \ldots, l-1), \quad \varphi_{l}(\sigma)=\frac{\sigma|\sigma|^{n-1}}{E_{l}} .
$$

Из условия совместности деформаций (19) и первых $(l-1)$ соотношений (32) имеем

$$
\sigma_{i}=\frac{E_{i} \sigma_{1}}{E_{1}}(i=\overline{2, l-1}) .
$$

Подставляя найденное значение $\sigma_{i}$ в (20), найдем

$$
\sigma_{l}=\frac{1}{\alpha_{l}}\left(\sigma_{0}-\frac{\sigma_{1}}{E_{1}} \sum_{i=1}^{l-1} \alpha_{i} E_{i}\right)
$$


Так как в процессе нагрузки все микронапряжения модели (16)-(19) положительны, то условие (21) при $i=l$ принимает вид

$$
\frac{\sigma_{1}^{0}}{E_{1}}=\frac{1}{E_{2}}\left(\frac{\sigma_{0}-\sigma_{1}^{0} \sum_{i=1}^{l} \frac{\alpha_{i} E_{i}}{E_{1}}}{\alpha_{l}}\right)^{n} .
$$

Из уравнения (34) очевидно, что $\sigma_{1}^{0}$ является корнем уравнения (неизвестная обозначена через $x$ )

$$
\frac{x}{E_{1}}=\frac{\left(\frac{\beta_{1}}{\alpha_{l}}\right)^{n}\left(\frac{\sigma_{0}}{\beta_{1}}-x\right)^{n}}{E_{2}}
$$

где

$$
\beta_{1}=\frac{1}{E_{1}}\left(\sum_{i=1}^{l-1} \alpha_{i} E_{i}\right)
$$

При конкретизации (32) уравнения (23) и (25) принимают вид

$$
\begin{aligned}
& \left\{\begin{array}{l}
\frac{\sigma_{1}^{*}}{E_{1}}+p_{1}^{*}=\frac{\sigma_{i}^{*}}{E_{i}}+p_{i}^{*} \quad(i=2,3, \ldots, l-1), \\
\frac{\sigma_{1}^{*}}{E_{1}}+p_{1}^{*}=\frac{\sigma_{l}^{*}\left|\sigma_{l}^{*}\right|^{n-1}}{E_{l}}+p_{l}^{*} ;
\end{array}\right. \\
& \left\{\begin{array}{l}
\frac{\Delta \sigma_{1}}{E_{1}}+p_{1}^{*}=\frac{\Delta \sigma_{i}}{E_{i}}+p_{i}^{*}(i=2,3, \ldots, l-1), \\
\frac{\Delta \sigma_{1}}{E_{1}}+p_{1}^{*}=\frac{\Delta \sigma_{l}\left|\Delta \sigma_{l}\right|^{n-1}}{E_{l}}+p_{l}^{*} .
\end{array}\right.
\end{aligned}
$$

Выражая из первых уравнений систем (37) и (38) величины $\sigma_{i}^{*}$ и $\Delta \sigma_{i}$ соответственно через $\sigma_{1}^{*}$ и $\Delta \sigma_{1}$ и подставляя их в (22) и (24), находим

$$
\begin{aligned}
& \sigma_{l}^{*}=\frac{1}{\alpha_{l}}\left[\sigma_{0}-\sigma_{1} \beta_{1}-\sum_{i=2}^{l-1}\left(p_{1}^{*}-p_{i}\right) \alpha_{i} E_{i}\right] ; \\
& \Delta \sigma_{l}=-\frac{1}{\alpha_{l}}\left[\Delta \sigma_{1} \beta_{1}+\sum_{i=2}^{l-1}\left(p_{1}^{*}-p_{i}\right) \alpha_{i} E_{i}\right],
\end{aligned}
$$

где $\beta_{1}$ определяется (36).

Вычитая из второго уравнения системы (37) второе уравнение системы (38), легко видеть, что разность $x=\sigma_{1}^{*}-\Delta \sigma_{1}$ является корнем уравнения

$$
\frac{x}{E_{1}}=\frac{1}{E_{2}}\left(\frac{\beta_{1}}{\alpha_{l}}\right)^{n}\left\{\left[\frac{\sigma_{0}}{\beta_{1}}-\Delta \sigma_{1}-\beta_{2}-x\right]^{n}+\left(\Delta \sigma_{1}+\beta_{2}\right) \cdot\left|\Delta \sigma_{1}+\beta_{2}\right|^{n-1}\right\},
$$

где $\beta_{2}=\frac{1}{\beta_{1}} \sum_{i=2}^{l-1}\left(p_{1}^{*}-p_{i}^{*}\right) \alpha_{i} E_{i}$.

Поскольку $\sigma_{1}^{0}$ и $\sigma_{1}^{*}-\Delta \sigma_{1}$ являются корнями различных уравнений (см. правые части (35) и (41) ), то условие (26), вообще говоря, не выполняется. Теорема доказана.

Выполним более детальное исследование уравнений (26), (35), (41).

СЛЕДСТВИЕ 1. Если $\Delta \sigma_{1}>\beta_{2}$, то при $n>1$ выполняется

а при $0<n<1$

$$
\varphi_{1}\left(\sigma_{1}^{0}\right)>\varphi_{1}\left(\sigma_{1}^{*}\right)-\varphi_{1}\left(\Delta \sigma_{1}\right)
$$

$$
\varphi_{1}\left(\sigma_{1}^{0}\right)<\varphi_{1}\left(\sigma_{1}^{*}\right)-\varphi_{1}\left(\Delta \sigma_{1}\right) .
$$

Д о к а 3 а т е л ь с т в о. Обозначим через $x_{1}$ корень уравнения (35), а через $x_{2}=\sigma_{1}^{*}-\Delta \sigma_{1}-$ корень уравнения (41). Величину $x_{1}$ можно определить, построив график функции $y=\frac{x}{E_{1}}$ и $y=\left(\frac{\beta_{1}}{\alpha_{l}}\right)^{n}\left(\frac{\sigma_{0}}{\beta_{1}}-x\right)^{n} \frac{1}{E_{2}}$ (см. рис.). 
Подставляя далее $x_{2}$ в (41) и учитывая, что $\Delta \sigma_{1}>\beta_{2}$ по условию, получим тождество

$$
\frac{x_{2}}{E_{1}}=\frac{1}{E_{2}}\left(\frac{\beta_{1}}{\alpha_{l}}\right)^{n}\left[\left(\frac{\sigma_{0}}{\beta_{1}}-\Delta \sigma_{1}-\beta_{2}-x_{2}\right)^{n}+\left(\Delta \sigma_{1}+\beta_{2}\right)^{n}\right] \text {. }
$$

Воспользовавшись тривиальным неравенством $a^{n}+b^{n}<<(a+b)^{n} \quad(a>0, b>0, n>1)$, из (44) имеем неравенство

$$
\frac{x_{2}}{E_{1}}<\left(\frac{\beta_{1}}{\alpha_{l}}\right)^{n}\left(\frac{\sigma_{0}}{\beta_{1}}-x_{2}\right)^{n} \frac{1}{E_{2}} .
$$

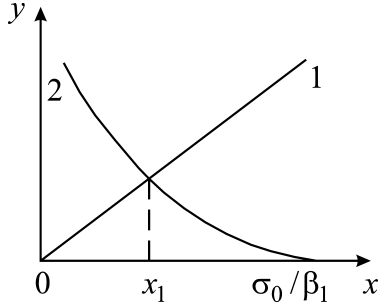

Графики функций: $1-y=x / E_{1}$;

$2-y=\left(\beta_{1} / \alpha_{l}\right)^{n}\left(\sigma_{0} / \beta_{1}-x\right)^{n} E_{2}^{-1}$

Значения $x_{2}$, для которых выполняется (45), находятся левее $x_{1}$ (см. рис.), т.е. $x_{2}<x_{1}$. Отсюда очевидно, что (42) выполняется. Используя затем тривиальное $a^{n}+b^{n}>(a+b)^{n},(a>0, b>0$, $0<n<1)$ из (44), получим

$$
\frac{x_{2}}{E_{1}}>\left(\frac{\beta_{1}}{\alpha_{l}}\right)^{n}\left(\frac{\sigma_{0}}{\beta_{1}}-x_{2}\right)^{n} \frac{1}{E_{2}},
$$

откуда следует $x_{2}>x_{1}$, и неравенство (43) выполняется. Следствие доказано.

СЛЕДСТВИЕ 2. Если $\Delta \sigma_{1}<\beta_{2}$, то при $n>1$ выполняется неравенство (43), а при $0<n<1-$ неравенство (42).

Д о к а з а т е л ь с т в о проводится аналогично доказательству следствия 1.

Таким образом, из теоремы 3 и следствий 1 и 2 следует, что эффект влияния деформации ползучести на величину упругой деформации возможен лишь для физически нелинейно упругого материала, при этом может происходить как упругое упрочнение материала (в смысле уменьшения упругих деформаций), так и его упругое разупрочнение за счет деформации ползучести. Для таких материалов классическое определение упругой деформации, функционально связывающей компоненты тензоров напряжений и деформаций, становится несправедливым, поскольку величина упругой деформации в процессе ползучести зависит от накопленной деформации ползучести. Причем этот эффект не имеет никакого отношения к подобным явлениям для стареющего материала, поскольку после разгрузки и полной релаксации остаточных напряжений $\Delta \sigma_{i}$ в локальных элементах структурной модели (при $t \rightarrow+\infty$ ) первоначальные упругие свойства материала полностью восстанавливаются.

\section{БИБЛИОГРАФИЧЕСКИЙ СПИСОК}

1. Работнов Ю. П. Ползучесть элементов конструкций. - М.: Наука, 1966. - 752 с.

2. Мейз Дж. Теория и задачи механики сплошных сред. - М.: Мир, 1974. - 284 с.

3. Радченко В. П., Еремин Ю. А. Реологическое деформирование и разрушение материалов и элементов конструкций. - М.: Машиностроение-1, 2004. - 264 с.

4. Гохфельд Д. А. Садаков О. С. Пластичность и ползучесть элементов конструкция при повторном нагружении. - М.: Машиностроение, 1984. - 256 с.

5. Зарубин В. С. Прикладные задачи термопрочности элементов конструкций. - М.: Машиностроение, 1985. — $294 \mathrm{c}$.

6. Новожилов В. В., Кадашевич Ю. И. Микронапряжения в конструкционных материалах. — М.: Машиностроение, Л. отд-ние, 1990. - 223 с.

7. Русинко К. Н. Теория пластичности и неустановившейся ползучести. — Львів: Вища школа, 1981. — 148 с.

8. Шевченко Ю. Н., Марина В. Ю. Структурная модель среды при неизотермическом процессе нагружения // Прикладная механика, 1976. — № 12. - С. 19-27.

9. Ванц X. Изменение механических свойств компактной костной ткани человека в зависимости от возраста // Механика полимеров, 1975. — № 4. - С. 659-663.

10. Кнетс И. В., Вилкс Ю. К. Ползучесть компактной костной ткани человека при растяжении // Механика полимеров, 1975. - № 4. - С. 634-638.

11. Мелнис А. Э., Лайзан Я. Б. Нелинейная ползучесть компактной костной ткани человека при растяжении // Механика полимеров, 1978. - № 1 - С. 97-100.

12. Самарин Ю. П. Уравнения состояния материалов со сложными реологическими свойствами. - Куйбышев: КуГУ, 1979. - $84 \mathrm{c.}$

13. Самарин Ю. П. Применение метода разделения деформации в теории ползучести / В сб.: Механика. Новые разработки конструкций. - Куйбышев: КПтИ, 1973. - С. 17-21.

14. Радченко В. П. Об одной структурной реологической модели нелинейно-упругого материала // Прикладная механика, 1990. - Т. 26, № 6. - С. 67-74. 
15. Радченко В. П., Самарин Ю. П. Влияние ползучести на величину упругой деформации слоистого композита // Механика композитных материалов, 1983. - № 2. - С. 231-237.

16. Самарин Ю. П. О применении теории управления к исследованию ползучести конструкций / В сб.: Механика деформируемых сред. - Куйбышев: КуГУ, 1976. — С. 123-129.

17. Радченко В. П., Самарин Ю. П. Влияние ползучести на обратимость упругой деформации статистически определимой стрежневой системы как целого / В сб.: Прочность и надежность конструкций. - Куйбышев: КуАИ, 1981. - С. 75-80.

18. Добелис М. А. Деформативные свойства деминерализованной компактной костной ткани человека при растяжении // Механика полимеров, 1978. — № 4. — С. 101-108.

Поступила 7.11.2006 г.

удк 539.376

\section{Н. Н. Попов, С. А. Забелин}

\section{РЕШЕНИЕ НЕЛИНЕЙНОЙ СТОХАСТИЧЕСКОЙ ЗАДАЧИ ПОЛЗУЧЕСТИ МЕТОДОМ МАЛОГО ПАРАМЕТРА ПРИ ПЛОСКОМ НАПРЯЖЕННОМ СОСТОЯНИИ}

Разработан аналитический метод решения плоской нелинейной задачи установившейся ползучести стохастически неоднородной среды. Стохастичность введена в определяющее соотношение ползучести, взятое в соответствии с нелинейной теорией вязкого течения. Применен метод разложения по малому параметру, при котором учитываются не только линейные, но и квадратичные члены. Вычислены дисперсии случайного поля напряжений. Произведено сравнение результатов, полученных в первом и во втором приближениях.

Аналитические методы решений стохастических краевых задач для структурно-неоднородных материалов хорошо разработаны для линейно упругих сред [1]. В условиях ползучести разработка аналитических методов решения стохастических задач сталкивается с серьезными трудностями, основными из которых являются физическая и статистическая нелинейность определяющих уравнений. Одним из основных методов решения стохастических задач является метод малого параметра, который относительно задач механики деформируемого твердого тела развивался в работах $[1,2]$. Суть этого метода состоит в том, что, разлагая компоненты тензоров напряжений и деформаций в ряд по малому параметру, статистически нелинейную задачу можно свести к последовательности статистически линейных задач. Однако этот подход связан с трудностями вычислительного характера, поэтому при решении конкретных стохастических задач ползучести обычно ограничиваются первым приближением, которое справедливо для слабонеоднородных сред [3-6].

В данной работе на основе второго приближения метода малого параметра приводится решение задачи о нелинейной ползучести стохастически неоднородной плоскости при двухосном растяжении. При этом вводятся ограничения о малости упругих деформаций, и считается, что ими допустимо пренебречь. Среда считается стохастически неоднородной, так что тензоры напряжений и деформаций являются случайными функциями координат $x_{1}, x_{2}$.

Пусть компоненты тензора напряжений $\sigma_{i j}$ удовлетворяют уравнениям равновесия

$$
\sigma_{i j, j}=0(i, j=1,2),
$$

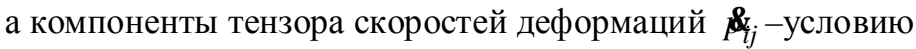

$$
\Lambda_{i j} \Lambda_{k l} \delta_{k, i l}=0 \text {, }
$$

которое получается из уравнения совместности для деформаций путем дифференцирования по времени, $\Lambda_{i j}$ - единичный антисимметричный псевдотензор

$$
\Lambda=\left(\begin{array}{cc}
0 & 1 \\
-1 & 0
\end{array}\right)
$$

По повторяющимся индексам производится суммирование от 1 до 2.

Уравнения (1) и (2) замыкаются определяющим соотношением, которое принимается в соответствии с нелинейной теорией вязкого течения (установившейся ползучести) в стохастической форме [3]: 